(1)

CrossMark

\title{
Patterns of regional lung physiology in cystic fibrosis using ventilation magnetic resonance imaging and multiple-breath washout
}

\author{
Laurie J. Smith $\mathbb{1}^{1,2}$, Guilhem J. Collier ${ }^{1}$, Helen Marshall ${ }^{1}$, Paul J.C. Hughes ${ }^{1}$, \\ Alberto M. Biancardi ${ }^{1}$, Martin Wildman ${ }^{3}$, Ina Aldag ${ }^{2}$, Noreen West ${ }^{2}$, \\ Alex Horsley $\mathbb{1}^{1,4}$ and Jim M. Wild ${ }^{1,5}$
}

Affiliations: ${ }^{1}$ POLARIS, Academic Radiology, University of Sheffield, Sheffield, UK. ${ }^{2}$ Sheffield Children's Hospital NHS Foundation Trust, Sheffield, UK. ${ }^{3}$ Sheffield Teaching Hospital NHS Foundation Trust, Sheffield, UK. ${ }^{4}$ Respiratory Research Group, Division of Infection, Immunity and Respiratory Medicine, University of Manchester, Manchester, UK. Insigneo, Institute of in-silico Medicine, Sheffield, UK.

Correspondence: Jim M. Wild, POLARIS, Academic Radiology, Dept of Infection, Immunity and Cardiovascular Disease, University of Sheffield, Sheffield, UK. E-mail: j.m.wildasheffield.ac.uk

@ERSpublications

Ventilation distribution on MRI improves at TLC and two distinct patterns of regional lung disease in CF are highlighted, where abnormal FEV 1 is associated with VDP $>10 \%$. Ventilation MRI and MBW are highly correlated. http://ow.ly/NvyS30lOP4O

Cite this article as: Smith LJ, Collier GJ, Marshall H, et al. Patterns of regional lung physiology in cystic fibrosis using ventilation magnetic resonance imaging and multiple-breath washout. Eur Respir J 2018; 52 : 1800821 [https://doi.org/10.1183/13993003.00821-2018].

ABSTRACT Hyperpolarised helium-3 ( $\left.{ }^{3} \mathrm{He}\right)$ ventilation magnetic resonance imaging (MRI) and multiple-breath washout (MBW) are sensitive methods for detecting lung disease in cystic fibrosis (CF). We aimed to explore their relationship across a broad range of CF disease severity and patient age, as well as assess the effect of inhaled lung volume on ventilation distribution.

32 children and adults with $\mathrm{CF}$ underwent $\mathrm{MBW}$ and ${ }^{3} \mathrm{He}-\mathrm{MRI}$ at a lung volume of end-inspiratory tidal volume (EIVT). In addition, 28 patients performed ${ }^{3} \mathrm{He}-\mathrm{MRI}$ at total lung capacity. ${ }^{3} \mathrm{He}-\mathrm{MRI}$ scans were quantitatively analysed for ventilation defect percentage (VDP), ventilation heterogeneity index (VHI) and the number and size of individual contiguous ventilation defects. From MBW, the lung clearance index, convection-dependent ventilation heterogeneity (Scond) and convection-diffusion-dependent ventilation heterogeneity (Sacin) were calculated.

VDP and VHI at EIVT strongly correlated with lung clearance index ( $\mathrm{r}=0.89$ and $\mathrm{r}=0.88$, respectively), Sacin ( $r=0.84$ and $r=0.82$, respectively) and forced expiratory volume in $1 \mathrm{~s}(\mathrm{FEV} 1)(\mathrm{r}=-0.79$ and $\mathrm{r}=-0.78$, respectively). Two distinct ${ }^{3} \mathrm{He}-\mathrm{MRI}$ patterns were highlighted: patients with abnormal FEV1 had significantly $(\mathrm{p}<0.001)$ larger, but fewer, contiguous defects than those with normal FEV1, who tended to have numerous small volume defects. These two MRI patterns were delineated by a VDP of $\sim 10 \%$. At total lung capacity, when compared to EIVT, VDP and VHI reduced in all subjects $(\mathrm{p}<0.001)$, demonstrating improved ventilation distribution and regions of volume-reversible and nonreversible ventilation abnormalities.

This article has supplementary material available from erj.ersjournals.com

Received: May 012018 | Accepted after revision: Sept 102018

Copyright CERS 2018 


\section{Introduction}

Hyperpolarised gas ventilation magnetic resonance imaging (MRI) allows for detailed and sensitive quantitative assessment of regional lung ventilation abnormalities in patients with obstructive airways disease [1-4]. The MRI technique usually involves the inhalation of a fixed volume of hyperpolarised noble gas and the resulting distribution of ventilation is imaged during a short breath-hold. In patients with cystic fibrosis (CF) it has been shown to be highly sensitive to detect early lung disease in children [5, 6], to track disease progression [7] and to assess treatment response [8]. However, the use of a fixed inhalation volume will result in patients with smaller lung volumes being closer to their total lung capacity (TLC) than patients with larger lung volumes. The effect that this has on the distribution of ventilation has not yet been assessed in CF. In addition, understanding the nature and pattern of regional ventilation defects seen on MRI, across the spectrum of CF lung disease, will allow for a greater understanding of lung disease pathophysiology and progression.

The lung clearance index (LCI), derived from multiple-breath washout (MBW) is a physiological measure of global ventilation heterogeneity that is sensitive to early lung disease [9]. LCI correlates with structural abnormalities seen on computed tomography [10-12] and MRI [13] and is also sensitive to treatment response [14]. The utility of LCI in CF has been established primarily in children and patients with mild disease. In more severe CF lung disease, when previously obstructed regions open up to tidal ventilation due to treatment, the LCI response is unpredictable and may worsen despite improvements in forced expiratory volume in $1 \mathrm{~s}$ (FEV1) [15-19]. Therefore, comparing LCI to ventilation MRI may help to better understand ventilation abnormalities expressed by the two methods.

In this work we aimed to 1) compare ventilation MRI and MBW, including LCI and the phase III slope outcomes Scond (convection-dependent ventilation heterogeneity) and Sacin (convection-diffusiondependent ventilation heterogeneity), in children and adults with CF with a broad range of lung disease severity; 2) assess ventilation MRI at two different lung volumes in order to investigate the nature of reversible airway obstruction in CF; and 3) assess the relationship between size and number of individual contiguous ventilation defects from ventilation MRI in CF patients across a broad range of disease severity to aid understanding of regional imaging data and disease status.

\section{Methods}

Children and adults with CF were recruited from three UK specialist centres (Sheffield Children's Hospital and Northern General Hospital, Sheffield and Manchester Adult CF Centre, Manchester). Patients were required to be aged $>5$ years, be clinically stable for 4 weeks prior to their visit and achieve an FEV1 $>30 \%$ predicted within the previous 6 months. This study was approved by the Yorkshire and the Humber Leeds West research ethics committee (16/YH/0339). Parents/guardians of children and all adult patients provided written informed consent.

\section{MRI acquisition}

Ventilation MRI was performed on a 1.5T GE HDx scanner (GE, Milwaukee, WI, USA) using hyperpolarised helium-3 $\left({ }^{3} \mathrm{He}\right)$ using a transmit-receive vest coil (CMRS, Milwaukee, WI, USA) and a three-dimensional (3D) ventilation imaging sequence as described previously [20]. Images were acquired at two different lung inhalation volumes during separate breath-holds. Firstly, images were acquired at a lung volume corresponding to end-inspiratory tidal volume (EIVT), by inhaling a predetermined fixed volume of test gas from their resting functional residual capacity (FRC). This corresponds to the approximate inspired volume most typically reported in studies to date $[1,5,6,8,21-28]$. The volume of gas was titrated based on the subject's height and consisted of scaled doses of ${ }^{3} \mathrm{He}$ and balanced with nitrogen (online supplementary table S1). Secondly, images were acquired at TLC by repeating the EIVT breathing manoeuvre, immediately followed by a full inhalation of room air. Further methodological details are described in the online supplementary material.

\section{MRI post-processing}

For both the EIVT and $\mathrm{TLC}{ }^{3} \mathrm{He}$ and ${ }^{1} \mathrm{H}$ image pairs, image metrics were calculated from a semi-automated segmentation [29]. The ${ }^{3} \mathrm{He}$ images were segmented in order to calculate the ventilated lung volume ( $\mathrm{Vv}$ ) and the ${ }^{1} \mathrm{H}$ images were used to calculate the thoracic cavity volume (TCV). From these two segmentations the ventilation defect percentage (VDP) and the ventilation heterogeneity index (VHI) were calculated (table 1 and online supplementary material).

Contiguous individual ventilation defects in $3 \mathrm{D}$ were assessed. Defects that contributed to $<1 \%$ of total VDP were discarded. The number of remaining defects as well as the volume of individual defects were calculated. The image analysis workflow is summarised in online supplementary figure S2. 
TABLE 1 Description of metrics calculated from helium-3 $\left({ }^{3} \mathrm{He}\right)$ and ${ }^{1} \mathrm{H}$ magnetic resonance imaging (MRI)

Thoracic cavity volume (TCV)

Ventilated volume (VV)

Ventilation defect percentage (VDP)

Ventilation heterogeneity index (VHI)

\section{Number of ventilation defects (ndefects)}

\section{Largest ventilation defect}

Reversible-volume index
Calculated from the segmentation of the ${ }^{1} \mathrm{H}$ anatomical MRI. The TCV is the lung volume at which ${ }^{3} \mathrm{He}-\mathrm{MRI}$ is performed and is measured in litres.

Calculated from ${ }^{3} \mathrm{He}$ ventilation image segmentation. VV represents the volume of ventilated lung and is measured in litres.

The percentage of the TCV that is not ventilated in the ${ }^{3} \mathrm{He}$ MRI images. Areas of the ${ }^{3} \mathrm{He}$ image that contribute to VDP appear black. It is calculated as VDP=100-((VV/TCV) $\times 100)$. Larger VDP values are associated with increased lung disease.

A marker of the heterogeneity of the ${ }^{3} \mathrm{He}$ signal within ventilated regions of the ${ }^{3} \mathrm{He} \mathrm{MRI}$ images. For each ventilated pixel, a local coefficient of variation of signal intensity in the surrounding pixels is computed. VH is the interquartile range of the distribution of those values. Increased VHI is associated with increased ventilation heterogeneity and therefore increased lung disease.

The number of individual three-dimensional contiguous ventilation defects within the subject's lung. This only includes unventilated lung areas contributing to VDP. Defects were counted if the defect volume was $>1 \%$ of total VDP.

This is the volume of the largest contiguous ventilation defect within the lungs. It is measured in litres and as a percentage of the TCV.

This represents the relative change that occurs in VV in response to the increase in TCV when comparing EIVT to TLC images. The reversible-volume index is $\geqslant 1.0$; the larger the value above 1.0 the greater the degree of EIVT ventilation defects that have resolved at TLC. In a healthy subject's lungs or in the lungs of a patient with nonreversible ventilation defects (resulting from complete obstruction), an increase in TCV due to deep inhalation will result in equal increase in $\mathrm{VV}$ and the reversible-volume index will be 1 . In contrast, any ventilation defect present at EIVT that at least partially resolves at TLC will produce a reversible-volume index $>1$ (online supplementary figure S3).

To describe the degree of ventilation change from EIVT to TLC, the reversible-volume index was calculated from the EIVT and TLC images using:

$$
\text { Reversible-volume index }=\frac{(\Delta \mathrm{VV})}{(\Delta \mathrm{TCV})}
$$

where $\Delta \mathrm{VV}=\mathrm{VV}_{(\mathrm{TLC})}-\mathrm{VV}_{(\mathrm{EIVT})}$ and $\Delta \mathrm{TCV}=\mathrm{TCV}_{(\mathrm{TLC})}-\mathrm{TCV}_{(\mathrm{EIVT})}$. Finally, the difference in VHI $(\Delta \mathrm{VHI})$ was quantified by $\Delta \mathrm{VHI}=\mathrm{VHI}_{(\mathrm{TLC})}-\mathrm{VHI}_{(\mathrm{EIVT})}$

\section{Pulmonary function}

MBW was performed as previously described using a modified open-circuit Innocor (Innovision, Glamsbjerg, Denmark) and $0.2 \%$ sulfur hexafluoride $\left(\mathrm{SF}_{6}\right)$ [30]. MBW was performed in triplicate, both seated and supine [31]. From MBW, the metrics LCI, Scond and Sacin were calculated and the average taken from at least two technically acceptable trials. Spirometry and body plethysmography were performed to international standards [32, 33] using a PFT Pro (Vyaire, Basingstoke, UK) and recommended reference equations [34]. All tests were performed on the same day. Either MBW or MRI was performed first, followed by the other. Spirometry was always performed last.

\section{Statistical analysis}

Metrics were assessed for normality using the Shapiro-Wilks test and expressed as either mean $\pm \mathrm{SD}$ or median (range). Patients were grouped into three groups: group 1 consisted of patients with normal FEV1 $(>-1.64 \mathrm{z}$-score) and normal LCI $(<7.4$ [30]); group 2 had normal FEV1 but abnormal LCI $(\geqslant 7.4)$; and group 3 had both abnormal FEV1 and LCI. Group comparisons were assessed using the Kruskal-Wallis test with Dunn's multiple comparisons test. As a result of this analysis, two refined groups are referred to throughout the results: those with normal FEV1 (z-score >-1.64) and those with abnormal FEV1 (z-score $\leqslant-1.64)$. Spearman's correlation analysis was performed to assess the relationship between metrics. In total, 13 metrics were considered; therefore, after Bonferroni adjustment [35], a p-value $<0.004$ was considered significant for correlation analysis. The Wilcoxon-signed rank test was used to assess the difference in MRI metrics between EIVT and TLC. All analyses were performed in GraphPad Prism (V7.0, San Diego, CA, USA). 
Results

32 patients with CF were recruited and assessed (17 (53\%) female). Patient demographics, lung function and MRI metrics are presented in table 2. Of the 32 patients studied, all but one child had visible ventilation abnormalities on ventilation MRI at EIVT. Online supplementary figure S4 shows representative ${ }^{3} \mathrm{He}$ images for all patients. $30(94 \%)$ patients had a VDP $>2 \%$ at EIVT, the upper value from healthy controls previously reported [6]. In contrast, 26 (81\%) patients had raised LCI and 14 (44\%) patients had abnormal FEV1. This resulted in six patients with normal FEV1 and LCI (group 1), 12 patients with normal FEV1 but abnormal LCI (group 2) and 14 patients with abnormal FEV1 and LCI (group 3). At EIVT, group 3 had significantly higher VDP, VHI, largest individual defect and a significantly lower total number of individual defects $(\mathrm{p}<0.001)$, than groups 1 and 2 . However, there was no significant difference between groups 1 and 2 for these metrics, although a trend towards higher VHI was seen in group 2 (figure 1). The only metric to significantly distinguish groups 1 and 2 was Scond ( $\mathrm{p}=0.03$ ).

Figure 2 demonstrates 3D ventilation MRI images at EIVT with contiguous ventilation defects highlighted; the examples shown are for a patient in group 2 and a patient in group 3.

\section{Correlations between lung function and MRI at EIVT}

VDP demonstrated significant correlation ( $\mathrm{p}<0.001$; figure 3 ) with LCI $(\mathrm{r}=0.89)$ and Sacin $(\mathrm{r}=0.84)$, but not Scond ( $\mathrm{r}=0.32)$; in addition, VDP correlated with residual volume (RV)/total lung capacity (TLC) ( $\mathrm{r}=0.80$ ) and FEV1 $(\mathrm{r}=-0.79)$. VHI demonstrated significant correlations with LCI $(\mathrm{r}=0.88)$ and Sacin $(\mathrm{r}=0.82)$, but not Scond ( $\mathrm{r}=0.46$; online supplementary figure S5), and with RV/TLC ( $\mathrm{r}=0.78)$ and FEV1 ( $\mathrm{r}=-0.78)$. Supine MBW results also demonstrated significant equivalent correlations and are documented in online supplementary table S2.

The volume of the largest defect correlated significantly $(\mathrm{p}<0.001)$ with VDP $(\mathrm{r}=0.97$; figure 4$)$, LCI $(\mathrm{r}=0.85)$, FEV1 $(\mathrm{r}=-0.80)$ and Sacin $(\mathrm{r}=0.79)$ and the number of defects demonstrated a significant correlation with VDP $(\mathrm{r}=-0.86)$, LCI $(\mathrm{r}-0.75), \mathrm{FEV}_{1}(\mathrm{r}=0.75)$ and Sacin $(\mathrm{r}=-0.62)$.

\section{Ventilation MRI comparison between EIVT and TLC}

Ventilation images at both EIVT and TLC were successfully acquired from 28 patients (table 3). Two patients were excluded due to acquisition errors and two patients could not successfully coordinate the TLC breathing manoeuvre. The median (range) TCV measured from ${ }^{1} \mathrm{H}$ MRI at EIVT was 78.2 (61.2-95.0)\% TCV at TLC, which was significantly correlated to the FRC/TLC ratio (a marker of lung

TABLE 2 Patient demographics, lung function and ventilation magnetic resonance imaging metrics at end-inspiratory tidal volume (EIVT)

\begin{tabular}{lcccc} 
& All patients & Group 1 & Group 2 & Group 3 \\
\hline Subjects n (\% female) & $32(53)$ & 6 & 12 & 14 \\
Age years & $16.7(6.4-43.1)$ & $10.1(6.4-16.5)$ & $12.7(8.3-17.4)$ & $29.9(14.9-43.1)^{*}, \#$ \\
Height cm & $156.2 \pm 17.7$ & $137.0 \pm 16.1$ & $153.7 \pm 16.3$ & $166.4 \pm 11.7^{*}$ \\
Weight kg & $49.7 \pm 18.4$ & $32.8 \pm 12.5$ & $45.1 \pm 15.1$ & $60.9 \pm 16.2^{*}$ \\
FEV1 z-score & $-1.8 \pm 2.03$ & $0.5 \pm 1.2$ & $-0.6 \pm 0.7$ & $-3.9 \pm 0.9^{*}, \#$ \\
RV/TLC \% & $33.9(17.8-52.6)$ & $23.5(19.9-26.5)$ & $24.9(17.8-35.3)$ & $47.7(31.6-52.6)^{*}, \#$ \\
LCI & $10.0(6.0-17.8)$ & $6.7(6.0-7.0)$ & $7.9(7.4-10.3)$ & $13.3(8.3-17.8)^{*, \#}$ \\
Scond & $0.07 \pm 0.03$ & $0.04 \pm 0.02$ & $0.08 \pm 0.02^{*}$ & $0.09 \pm 0.03^{*}$ \\
Sacin & $0.14(0.04-0.55)$ & $0.08(0.04-0.10)$ & $0.10(0.05-0.19)$ & $0.30(0.14-0.55)^{*}, \#$ \\
LClsupine & $9.6(6.2-20.2)$ & $6.9(6.2-8.3)$ & $9.1(7.0-10.6)$ & $14.1(7.7-20.2)^{*, \#}$ \\
VDP \% & $14.9(0.2-45.0)$ & $2.7(0.2-3.3)$ & $4.2(1.5-9.2)$ & $29.0(9.5-45.0)^{*, \#}$ \\
VHI \% & $15.1(6.7-22.2)$ & $8.9(6.7-11.3)$ & $12.1(9.3-17.8)$ & $20.1(15.0-22.2)^{*}, \#$ \\
Largest defect \%TCV & $3.1(0.03-26.8)$ & $0.7(0.03-1.3)$ & $1.2(0.1-3.5)$ & $15.2(3.8-26.8)^{*}, \#$ \\
ndefects & $7(2-24)$ & $16(9-18)$ & $13(4-24)$ & $3(2-7)^{*, \#}$
\end{tabular}

Data are presented as $\mathrm{n}$, median (range) or mean \pm SD, depending on the distribution of individual metrics. Results are displayed for the whole population and for the three groups of patients. Group 1: patients with normal spirometry and lung clearance index (LCI); group 2: patients with normal spirometry but abnormal $\mathrm{LCl}$; group 3: abnormal spirometry and LCl. FEV1: forced expiratory volume in $1 \mathrm{~s}$; RV: residual volume; TLC: total lung capacity; Scond: convection-dependent ventilation heterogeneity; Sacin: convectiondiffusion-dependent ventilation heterogeneity; VDP: ventilation defect percentage; VHI: ventilation heterogeneity index; TCV: thoracic cavity volume; ndefects: number of defects. ${ }^{*}: p<0.05$ versus group 1; ${ }^{\#}$ : $p<0.05$ versus group 2 . 
a)

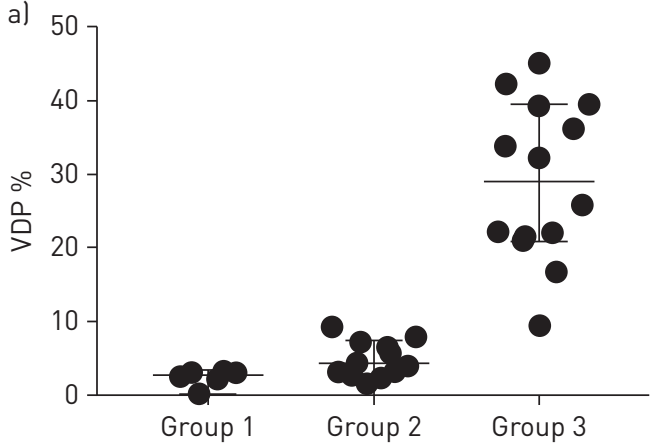

c)

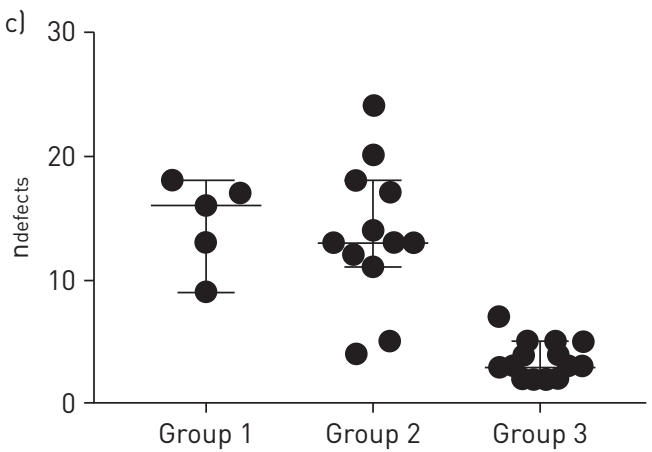

b)
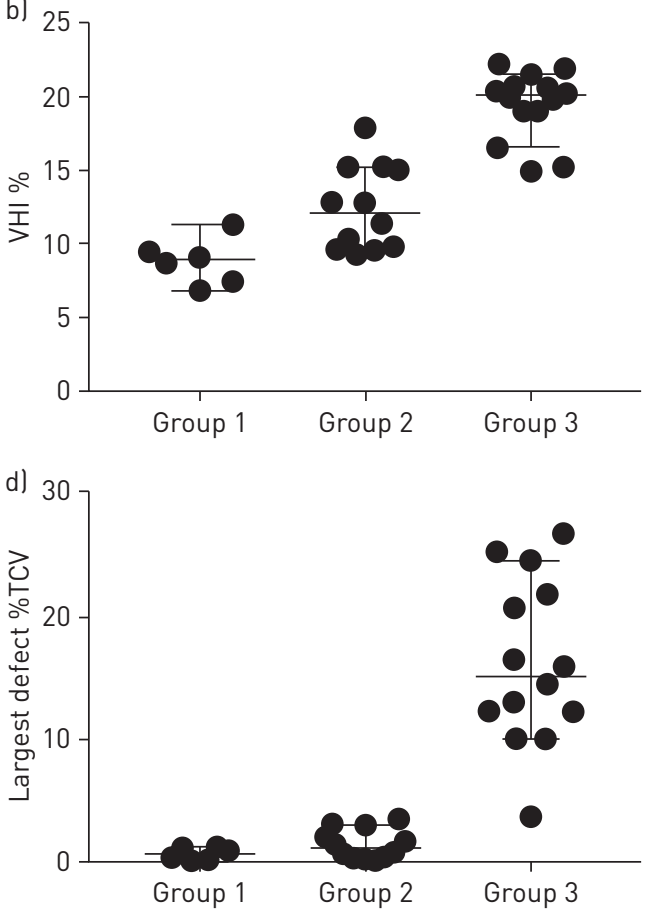

FIGURE 1 Kruskal-Wallis group comparison for a) ventilation defect percentage (VDP); b) ventilation heterogeneity index (VHI); c) number of defects (ndefects); and d) largest individual contiguous defect, calculated from ventilation magnetic resonance imaging at end-inspiratory tidal volume. Group 1 have both normal forced expiratory volume in $1 \mathrm{~s}\left(\mathrm{FEV}_{1}\right)$ and lung clearance index $(\mathrm{LCl})$; group 2 have normal $\mathrm{FEV} 1$, but abnormal LCl; group 3 have abnormal FEV1 and LCl. In all graphs, group 3 is significantly different when compared to groups 1 and $2(p<0.001)$, but there was no statistically significant difference between groups 1 and 2. TCV: thoracic cavity volume.

hyperinflation) measured during body plethysmography ( $\mathrm{r}=0.68)$. The TCV at TLC was 97.7 (85.0-107.7)\%TLC measured during body plethysmography.

At TLC there was a marked reduction in ventilation abnormalities. Signal intensity in ventilated regions of the lungs appeared more homogeneous, and in most patients some areas of unventilated lung became ventilated at TLC. This resulted in fewer ventilation defects at TLC for some patients, while in others ventilation abnormalities remained (figure 5). At TLC, when compared to EIVT, there was a significant decrease $(\mathrm{p}<0.001$; figure 6$)$ in MRI markers (expressed as median difference ( $95 \%$ confidence interval)), including VDP $-4.7(-11.0--2.2) \%$, VHI $-4.1 \quad(-5.6--3.1) \%$, volume of the largest defect -47.3 $(-160.1--17.1) \mathrm{mL}$ and largest defect expressed as a percentage of TCV $-1.7(-4.3--0.8) \% \mathrm{TCV} .10$ out of 28 patients had a reduction in the number of remaining defects at TLC $(\mathrm{p}=0.2)$, all of whom had normal FEV1. The reversible-volume index, but not $\Delta$ VHI significantly correlated with VDP at EIVT $(\mathrm{r}=0.85)$ and with LCI $(\mathrm{r}=0.82$; online supplementary figure S6), Sacin $(\mathrm{r}=0.75)$ and FEV1 $(\mathrm{r}=-0.74)$. The reversible-volume index was significantly higher in group 3 than in groups 1 and $2(p<0.001)$, but not between groups 1 and 2. $\Delta$ VHI was not significantly different between groups.

VDP at TLC significantly correlated with LCI $(r=0.85)$, Sacin $(r=0.77)$, FEV1 $(r=-0.79)$ and RV/TLC $(\mathrm{r}=0.86)$. VHI at TLC significantly correlated with LCI $(\mathrm{r}=0.82)$, Sacin $(\mathrm{r}=0.74)$, FEV1 $(\mathrm{r}=-0.84)$ and $\mathrm{RV} / \mathrm{TLC}(\mathrm{r}=0.86)$.

\section{Discussion}

In this study we present a detailed analysis of the relationship between MBW and hyperpolarised gas ventilation MRI in patients with $\mathrm{CF}$, across a broad range of age and disease severity. This analysis confirms the strong relationships between global MRI and MBW metrics that have been reported previously in smaller cohort studies, performed across narrower ranges of age and disease severity [5-7]. Previous work has documented that patients with CF have ventilation defects evident on hyperpolarised gas MRI $[1,8,21,24,36,37]$, that the technique is reproducible and repeatable $[21,24]$ and that it can be used to assess regional response to treatment $[8,28]$. Here we demonstrate what appear to be two distinct ventilation MRI patterns of CF lung disease: 1) patients who have numerous smaller defects (and normal 

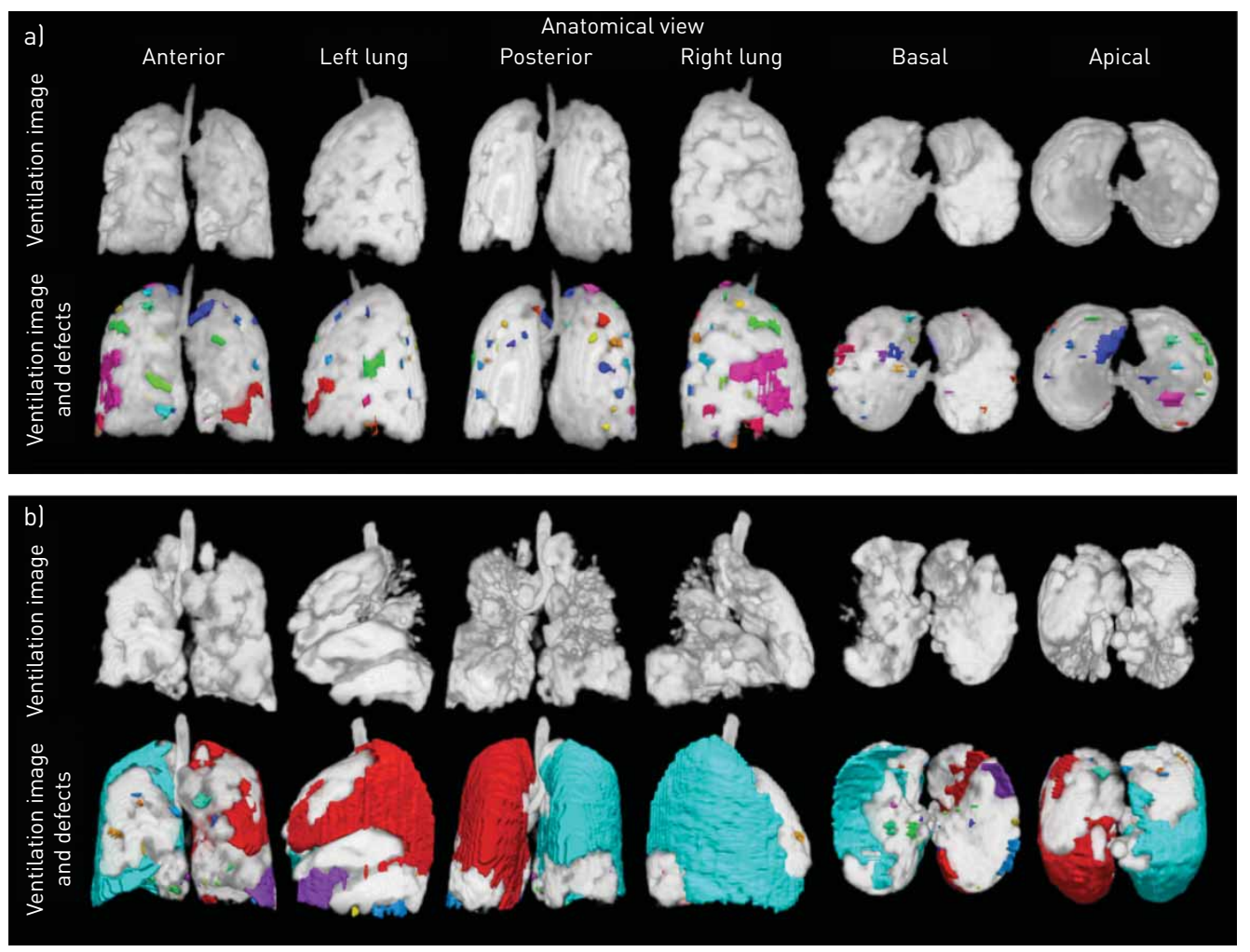

FIGURE 2 Demonstration of representative ventilation images at end-inspiratory tidal volume (EIVT) from a patient from a) group 2 and b) group 3. For both patients, the first row is a three-dimensional (3D) ventilation image and the second row is a $3 \mathrm{D}$ ventilation image with the segmented individual contiguous ventilation defects added in colour. The colours associated with defects are arbitrary, but each different colour represents a single different radiologically contiguous defect. For each patient the ventilation image is observed from various anatomical angles. a) Ventilation defect percentage (VDP) $5.4 \%$; largest defect (pink) $1.5 \%$ of the thoracic cavity volume (TCV); defects $n=18$; lung clearance index (LCI) 7.7; forced expiratory volume in $1 \mathrm{~s}$ (FEV1) $\mathrm{z}$-score -0.5 ; b) VDP $45.0 \%$; largest defect (red) $26.8 \%$ of the TCV; defects $\mathrm{n}=3$; LCl 14.9 ; FEV1 z-score -5.4 .

FEV1); and 2) patients who have fewer, but much larger contiguous defects, where FEV1 is invariably reduced. In addition, we have demonstrated that many of these ventilation abnormalities are lung-volume dependent. When larger volumes are inhaled, some apparently obstructed airways open and allow gas to ventilate previously unventilated areas.

This last observation has important implications from an imaging methodology perspective. Studies utilising hyperpolarised gas are often performed by inhaling fixed gas volumes, with $1 \mathrm{~L}$ of gas inhaled from FRC being common [21-25]. However, this results in smaller subjects being closer to their TLC than taller subjects, potentially reducing VDP and making cross-sectional comparisons challenging. Recent paediatric studies have titrated the inhaled volume based on measured or predicted lung volume $[6,7,26,27]$, and we recommend this practice for all patients with CF. However, it is important to note that we have not directly compared the inhaled volume given in this study to a fixed 1-L inhalation.

The finding that only some ventilation defects decrease in size at TLC implies that regions of volume-reversible airways obstruction and regions of complete airways obstruction (that are fixed on the timescale of the imaging session) co-exist in CF lungs. The reversibility of defects with deep inhalation highlights the probable value of physiotherapy and exercise in opening these lung regions, and also the potential for ventilation MRI to aid targeted therapies to be applied to specific lung regions. The change in ventilation from EIVT to TLC was not uniform across the population, with ventilation in some patients remaining distinctly abnormal at TLC. Assessing the transient nature of these lung-volume-dependent ventilation defects longitudinally may provide insight into the progression of disease pathophysiology.

The analysis of contiguous ventilation defects allows quantification and tracking of individual defects over time. Two distinct ventilation MRI patterns at EIVT are highlighted, which may represent different ends of the disease spectrum in CF. Figure 4 demonstrates that in those patients with normal FEV1, VDP is $<10 \%$ and predominantly consists of numerous small-volume defects (possibly due to predominantly peripheral 

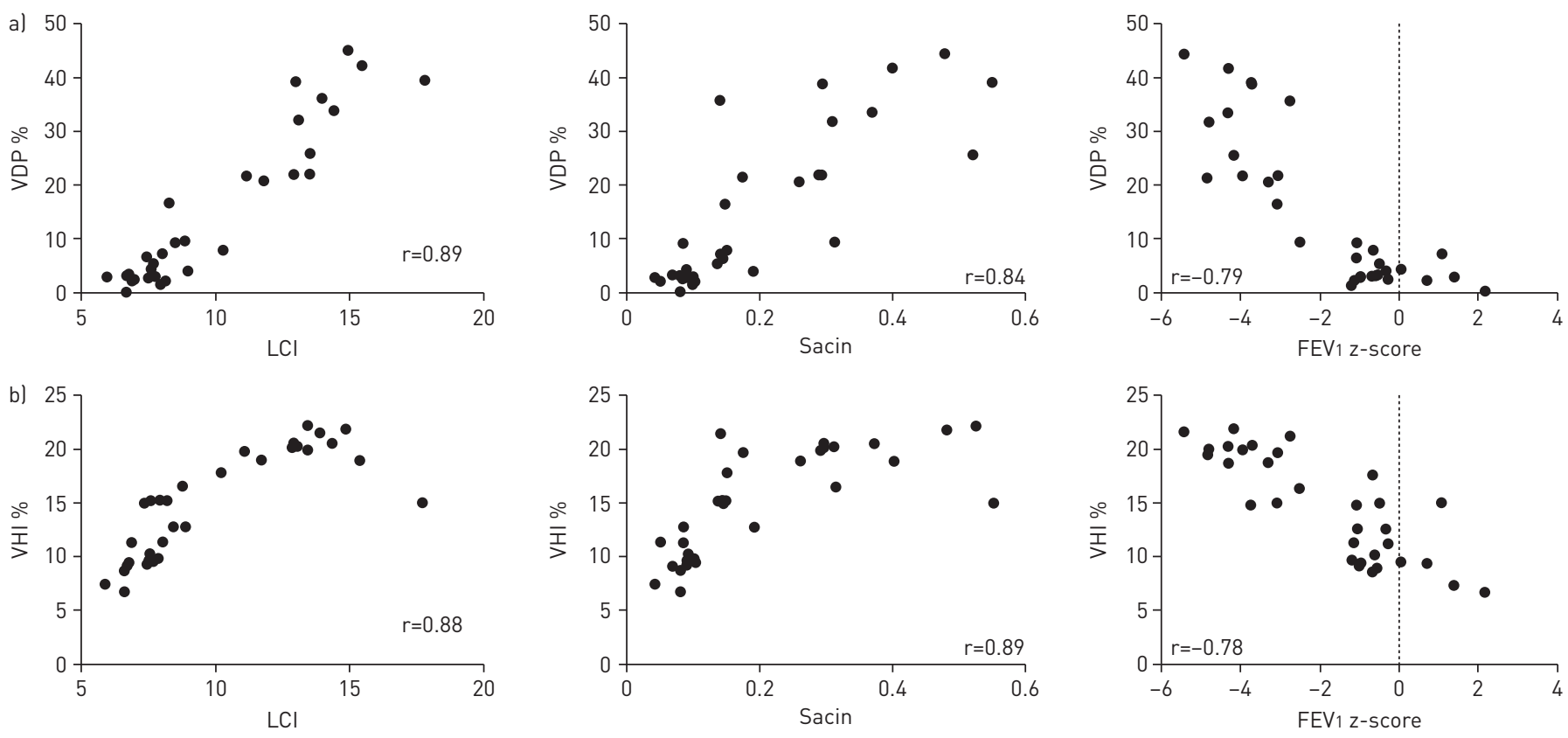

FIGURE 3 Scatter plots of a) ventilation defect percentage (VDP) and b) ventilation heterogeneity index (VHI), both performed at end-inspiratory tidal volume, against pulmonary function metrics, with Spearman correlation values ( $p<0.001$ in all). LCl: lung clearance index; Sacin: convectiondiffusion-dependent ventilation heterogeneity; FEV1: forced expiratory volume in $1 \mathrm{~s}$.

a)
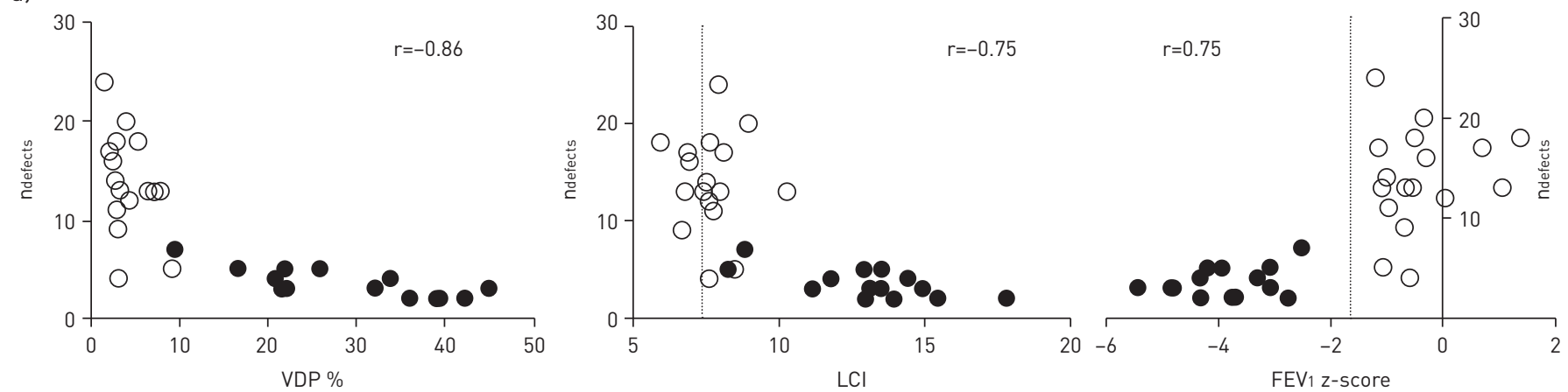

b)
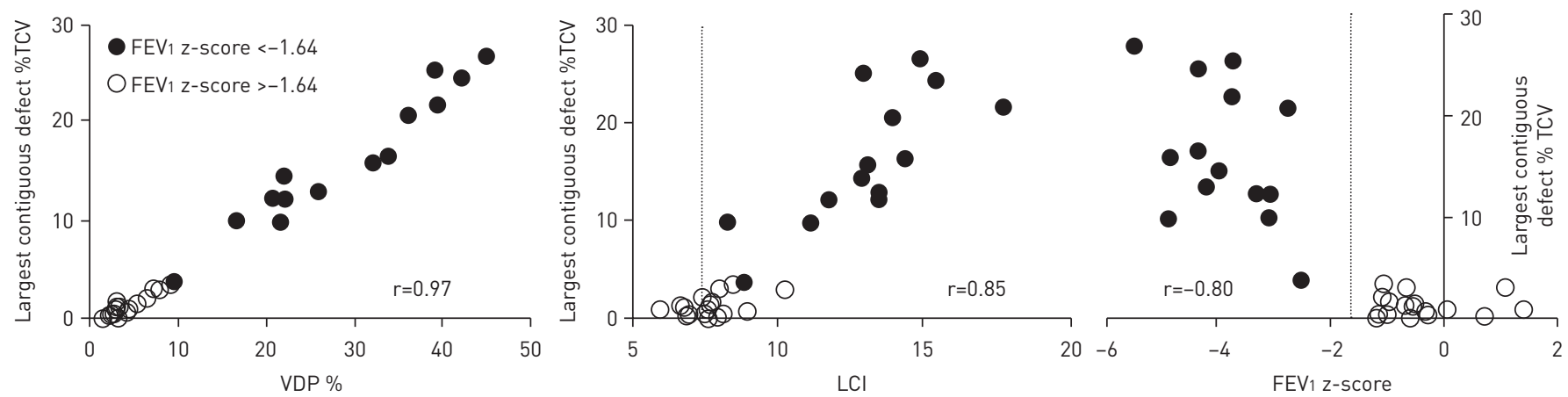

FIGURE 4 Scatter plots of a) the number of individual contiguous ventilation defects (ndefects) and the b) size of the largest individual contiguous defect at end-inspiratory tidal volume (EIVT) against ventilation defect percentage (VDP) at EIVT, lung clearance index (LCI) and forced expiratory volume in $1 \mathrm{~s}\left(\mathrm{FEV}_{1}\right)$. In each graph patients are divided into those with an FEV 1 z-score $<-1.64$ or $>-1.64$. For FEV 1 the dashed line represents the lower limit of normal $(-1.64)$ and for $\mathrm{LCl}$ the dashed line represents the upper limit of normal (7.4). All Spearman correlations have a p-value $<0.001$ 
TABLE 3 Ventilation magnetic resonance imaging metrics for the 28 patients with images acquired at both end-inspiratory tidal volume (EIVT) and total lung capacity (TLC)

EIV

$8.5(1.5-45.0)$

$15.2(7.4-22.2)$

$3.3(0.1-26.8)$

$9(2-24)$

TLC

$\begin{array}{lc}\text { VDP \% } & 8.5(1.5-45.0) \\ \text { VHI \% } & 15.2(7.4-22.2) \\ \text { Largest defect size \%TCV } & 3.3(0.1-26.8) \\ \text { ndefects } & 9(2-24)\end{array}$

Reversible-volume index $\Delta \mathrm{VHI}$

Data are presented as median (range). VDP: ventilation defect percentage; VHI: ventilation heterogeneity index; TCV: thoracic cavity volume; ndefects: number of defects. *: $p<0.05$ between lung volumes.

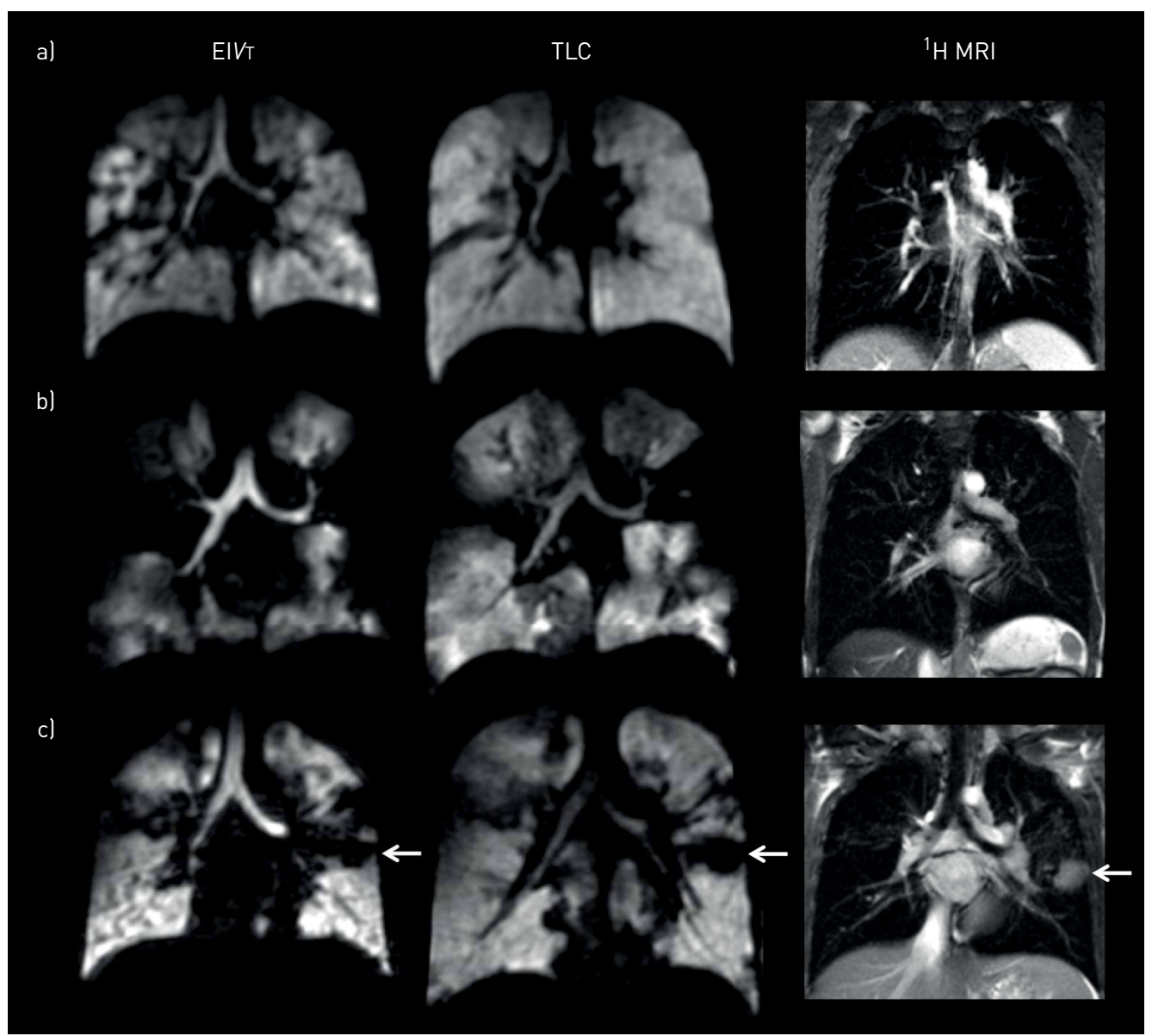

FIGURE 5 Representative helium-3 $\left({ }^{3} \mathrm{He}\right)$ ventilation magnetic resonance imaging (MRI) images acquired at end-inspiratory tidal volume (EIVT) and total lung capacity (TLC) from three subjects, with representative ${ }^{1} \mathrm{H}$ MRI. a) Forced expiratory volume in $1 \mathrm{~s}\left(F E V_{1}\right)$ z-score -0.5, lung clearance index (LCI) 7.7, residual volume (RV)/total lung capacity (TLC) $22.4 \%$. This subject has ventilation defects present at EIVT lventilation defect percentage (VDP) 5.4\%), which largely disappear at TLC (VDP 0.4\%), reversible-volume index 1.2; b) FEV1 z-score -3.7, LCl 17.8, RV/TLC 50.3\%. This subject has ventilation defects present at EIVT (VDP 39.5\%), some of which remain at TLC (VDP 18.2\%), reversible-volume index 2.0; c) FEV1 z-score $-1.1, \mathrm{LCl} 7.4, \mathrm{RV} / \mathrm{TLC}$ $24.8 \%$. This subject has ventilation defects present at EIVT (VDP 6.5\%), which largely remain at TLC (VDP $5.3 \%$ ) and therefore has a low reversible-volume index (1.0). The cause of part of the nonreversible ventilation can be seen in the left lung on the ${ }^{1} \mathrm{H}$ image where an area of significant mucus is present, corresponding to the ventilation defect seen on both EIVT and TLC ventilation images (arrow). 

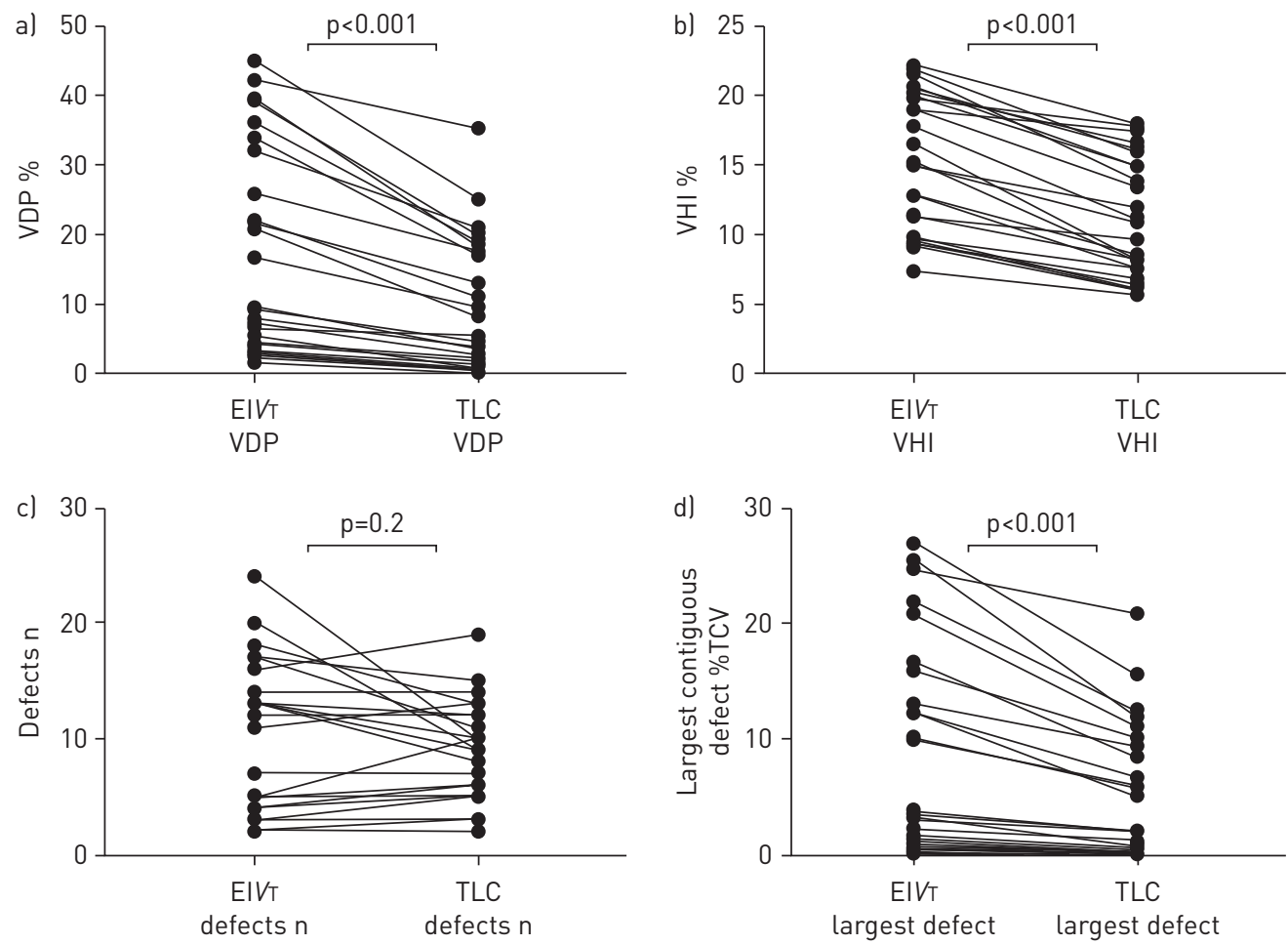

FIGURE 6 A comparison of ventilation magnetic resonance imaging metrics between end-inspiratory tidal volume (EIVT) and total lung capacity (TLC). At TLC there was a significant reduction in a) ventilation defect percentage (VDP), b) ventilation heterogeneity index (VHI) and d) the largest individual contiguous defect $(p<0.001) ; c)$ the number of defects declined only for some subjects with normal forced expiratory volume in $1 \mathrm{~s}$ (10 out of 28 patients). TCV: thoracic cavity volume.

airways disease), which also appear more likely to be reversible. However, by the time FEV1 becomes abnormal, VDP is invariably $>10 \%$. In these patients, VDP is dominated by fewer larger contiguous defects (suggesting widespread airways disease and lobar destruction), possibly in part due to smaller defects merging with disease progression. In addition, these larger defects are less likely to disappear with full inspiration, suggesting that a significant proportion of peripheral lung is not being routinely ventilated during tidal breathing. Such regions are likely to harbour reservoirs of trapped mucus and inflammatory exudate, encouraging further lung inflammation and damage. These findings in part are in contrast to previous work [37-39], which reported that the number of ventilation defects increased with worsening lung disease. This is probably due to the two-dimensional assessment of ventilation images in previous studies, resulting in individual defects that span multiple slices being classed as multiple defects, whereas in this $3 \mathrm{D}$ assessment of ventilation defects, defects are shown to be contiguous and not independent between slices, resulting in fewer individual defects.

There is an intuitive relationship between ventilation MRI and MBW metrics, as they both assess the distribution of inhaled gas within the lungs. These data suggest that this relationship is stronger when MRI was performed at EIVT when compared to TLC, due to the EIVT manoeuvre most closely representing the end-inspiratory cycle of quiet breathing performed during MBW. Ventilation MRI has the advantage that the exact regional nature of this ventilation distribution can be assessed, including lung regions that are entirely blocked (and hence silent to MBW testing). In this cohort we found that LCI and Sacin had strong relationships with VDP and VHI and also with reversible-volume index. However, Scond showed poor correlations due to the "plateau effect" evident in online supplementary figure S5, which occurs with increasing disease. This plateau has been reported previously [40], and suggests that Scond is useful primarily as a marker of very early CF lung disease, highlighted by the finding that Scond was the only metric to significantly differentiate between groups 1 and 2 . Therefore, convection-dependent ventilation heterogeneity seems to be an early event in disease progression. Figure 3 suggests that a similar relationship may be evident when comparing LCI and VHI, although the plateau effect for VHI occurs at much higher levels of LCI. Up to this point, VHI is strongly associated with increasing LCI. It is possible that with increasing lung disease in an individual, areas of increased VHI become nonventilated and contribute directly to VDP instead. 
Higher values for the reversible-volume index indicate that a greater proportion of EIVT ventilation defects receive ventilation at TLC, implying volume-reversible airway obstruction. Reversible-volume index showed significant correlations with both LCI and Sacin. We hypothesise that this positive correlation may indicate that ventilation defects present at EIVT, which become ventilated at TLC, may be lung regions responsible for delayed gas washout during MBW. Conversely, patients with large VDP but relatively low LCI may be explained by the presence of defects that are unable to achieve ventilation at TLC (i.e. low reversible-volume index), therefore these defects may not significantly contribute to the dynamic LCI signal (online supplementary figures S6 and S7). Therefore, the reversible-volume index may help explain why an unpredictable LCI response is seen with treatments in more severe and acute CF lung disease, despite clinical and spirometric improvements. We postulate that a lower reversible-volume index in a subject with significant ventilation abnormalities will result in a relatively low LCI for their level of lung disease (online supplementary figure S7). In response to treatment, we hypothesise that both the reversible-volume index and the LCI would increase in this case, caused by the opening of previously blocked lung regions to the MBW signal.

There are limitations to this study that require consideration. Whilst we have reported large numbers of patients for a ventilation MRI study $[1,5,6,8,21,22,26,27,36,37]$, there are still relatively small numbers of subjects in each subgroup, which inevitably limits the generalisability of this comparison. In comparing ventilation MRI with $\mathrm{MBW}$ we also acknowledge that the inert gases used have different diffusivity within the lung. ${ }^{3} \mathrm{He}$ has higher diffusivity in air when compared to $\mathrm{SF}_{6}$, suggesting $\mathrm{SF}_{6}$ may reveal larger ventilation defects if used in MRI. This has been reported when comparing ${ }^{3} \mathrm{He}$ with xenon-129 ventilation imaging in chronic obstructive pulmonary disease [41]. A limitation of the individual defect analysis is that it assesses contiguous areas of signal void within the ventilation images, which are not necessarily anatomically contiguous. The larger defects evident in more severe disease can in some cases be seen to merge across different lung lobes which are fed by distinct conducting airways and therefore do not represent a physiologically discrete defect caused by blockage of a single airway. Finally, in order to validate these cross-sectional findings, longitudinal data are required to determine whether the different patterns of ventilation observed behave as we predict on an individual basis over time.

In conclusion, this work adds to a growing body of work highlighting the use of ventilation MRI in CF, and specifically the role of VDP as a potential clinical tool and end-point in clinical trials. In particular two key novel aspects are highlighted that help define the clinical meaning and utility of the methodology. Firstly, we highlight a VDP value of $10 \%$, which separates normal and abnormal FEV1 values (the current clinical gold standard) and appears to delineate a boundary between the two distinct ventilation imaging patterns described, i.e. numerous small defects versus fewer large defects. Secondly, we demonstrate the coexistence of reversible and nonreversible regions of airway obstruction in CF using ventilation imaging at different lung volumes. This has direct impact on the longitudinal monitoring of an individual's lung health and delivery of regionally specific treatment.

Acknowledgements: The authors would like to acknowledge all members of the POLARIS research group at the University of Sheffield for their support. In particular, we would like to thank Leanne Armstrong for administrative support, Jody Bray for assisting with MRI scanning, Oliver Rodgers for polarisation of ${ }^{3} \mathrm{He}$ during MRI and Chris Johns for radiological review of all images. We would also like to thank the Cystic Fibrosis clinical teams at Sheffield Children's Hospital, Sheffield Teaching Hospital and Manchester CF Centre for their support. Finally, we would like to thank all of the participants for their time in taking part in this research.

Conflict of interest: L.J. Smith reports grants from the National Institute for Health Research (clinical doctoral research fellowship), during the conduct of the study. G.J. Collier has nothing to disclose. H. Marshall has nothing to disclose. P.J.C. Hughes has nothing to disclose. A.M. Biancardi has nothing to disclose. M. Wildman has nothing to disclose. I. Aldag has nothing to disclose. N. West has nothing to disclose. A. Horsley reports grants from the National Institute of Health Research (NIHRCS12-013), personal fees from Celtaxys Pharmaceuticals (for being an advisor), personal fees from Vertex Pharmaceuticals (for educational activities and advisory board), personal fees from Boehringer Ingelheim and Chiesi Ltd (both for advisory boards), nonfinancial support from Innovision ApS (collaboration agreement), grants from the Danish Government (Markedsmodingsfonden scheme for use of LCI system), and grants from the Cystic Fibrosis Trust and the Cystic Fibrosis Foundation, all outside the submitted work. J.M. Wild has nothing to disclose.

Support statement: This report is independent research supported by the National Institute for Health Research (NIHR-RP-R3-12-027 and ICA-CDRF-2015-01-027) and Health Education England and also the Medical Research Council (MR/M008894/1). The views expressed in this publication are those of the author(s) and not necessarily those of the NHS, the National Institute for Health Research, Health Education England or the Dept of Health. Funding information for this article has been deposited with the Crossref Funder Registry.

\section{References}

1 McMahon CJ, Dodd JD, Hill C, et al. Hyperpolarized ${ }^{3}$ helium magnetic resonance ventilation imaging of the lung in cystic fibrosis: comparison with high resolution CT and spirometry. Eur Radiol 2006; 16: 2483-2490. 
2 Kirby M, Svenningsen S, Owrangi A, et al. Hyperpolarized ${ }^{3} \mathrm{He}$ and ${ }^{129} \mathrm{Xe}$ MR imaging in healthy volunteers and patients with chronic obstructive pulmonary disease. Radiology 2012; 265: 600-610.

3 Svenningsen S, Guo F, McCormack DG, et al. Noncystic fibrosis bronchiectasis: regional abnormalities and response to airway clearance therapy using pulmonary functional magnetic resonance imaging. Acad Radiol 2017; 24: 4-12.

4 Svenningsen S, Kirby M, Starr D, et al. What are ventilation defects in asthma? Thorax 2014; 69: 63-71.

5 Kanhere N, Couch MJ, Kowalik K, et al. Correlation of lung clearance index with hyperpolarized ${ }^{129}$ Xe magnetic resonance imaging in pediatric subjects with cystic fibrosis. Am J Respir Crit Care Med 2017; 196: 1073-1075.

6 Marshall H, Horsley A, Taylor CJ, et al. Detection of early subclinical lung disease in children with cystic fibrosis by lung ventilation imaging with hyperpolarised gas MRI. Thorax 2017; 72: 760-762.

7 Smith L, Marshall H, Aldag I, et al. Longitudinal assessment of children with mild cystic fibrosis using hyperpolarized gas lung magnetic resonance imaging and lung clearance index. Am J Respir Crit Care Med 2018; 197: 397-400.

8 Altes TA, Johnson M, Fidler M, et al. Use of hyperpolarized helium-3 MRI to assess response to ivacaftor treatment in patients with cystic fibrosis. J Cyst Fibros 2017; 16: 267-274.

9 Aurora P, Gustafsson P, Bush A, et al. Multiple breath inert gas washout as a measure of ventilation distribution in children with cystic fibrosis. Thorax 2004; 59: 1068-1073.

10 Gustafsson PM, De Jong PA, Tiddens HA, et al. Multiple-breath inert gas washout and spirometry versus structural lung disease in cystic fibrosis. Thorax 2008; 63: 129-134.

11 Owens CM, Aurora P, Stanojevic S, et al. Lung clearance index and HRCT are complementary markers of lung abnormalities in young children with CF. Thorax 2011; 66: 481-488.

12 Ramsey KA, Rosenow T, Turkovic L, et al. Lung clearance index and structural lung disease on computed tomography in early cystic fibrosis. Am J Respir Crit Care Med 2016; 193: 60-67.

13 Stahl M, Wielpütz MO, Graeber SY, et al. Comparison of lung clearance index and magnetic resonance imaging for assessment of lung disease in children with cystic fibrosis. Am J Respir Crit Care Med 2017; 195: 349-359.

14 Amin R, Subbarao P, Lou W, et al. The effect of dornase alfa on ventilation inhomogeneity in patients with cystic fibrosis. Eur Respir J 2011; 37: 806-812.

15 Pfleger A, Steinbacher M, Schwantzer G, et al. Short-term effects of physiotherapy on ventilation inhomogeneity in cystic fibrosis patients with a wide range of lung disease severity. J Cyst Fibros 2015; 14: 627-631.

16 Welsh L, Nesci C, Tran H, et al. Lung clearance index during hospital admission in school-age children with cystic fibrosis. J Cyst Fibros 2014; 13: 687-691.

17 Horsley AR, Davies JC, Gray RD, et al. Changes in physiological, functional and structural markers of cystic fibrosis lung disease with treatment of a pulmonary exacerbation. Thorax 2013; 68: 532-539.

18 Robinson PD, Cooper P, Van Asperen P, et al. Using index of ventilation to assess response to treatment for acute pulmonary exacerbation in children with cystic fibrosis. Pediatr Pulmonol 2009; 44: 733-742.

19 Gozal D, Bailey SL, Keens TG. Evolution of pulmonary function during an acute exacerbation in hospitalized patients with cystic fibrosis. Pediatr Pulmonol 1993; 16: 347-353.

20 Horn FC, Tahir BA, Stewart NJ, et al. Lung ventilation volumetry with same-breath acquisition of hyperpolarized gas and proton MRI. NMR Biomed 2014; 27: 1461-1467.

21 Kirby M, Svenningsen S, Ahmed H, et al. Quantitative evaluation of hyperpolarized helium-3 magnetic resonance imaging of lung function variability in cystic fibrosis. Acad Radiol 2011; 18: 1006-1013.

22 Mentore K, Froh DK, de Lange EE, et al. Hyperpolarized HHe 3 MRI of the lung in cystic fibrosis: assessment at baseline and after bronchodilator and airway clearance treatment. Acad Radiol 2005; 12: 1423-1429.

23 O'Sullivan B, Couch M, Roche JP, et al. Assessment of repeatability of hyperpolarized gas MR ventilation functional imaging in cystic fibrosis. Acad Radiol 2014; 21: 1524-1529.

24 Paulin GA, Svenningsen S, Jobse BN, et al. Differences in hyperpolarized ${ }^{3} \mathrm{He}$ ventilation imaging after 4 years in adults with cystic fibrosis. J Magn Reson Imaging 2015; 41: 1701-1707.

25 Sun Y, O'Sullivan BP, Roche JP, et al. Using hyperpolarized ${ }^{3} \mathrm{He}$ MRI to evaluate treatment efficacy in cystic fibrosis patients. J Magn Reson Imaging 2011; 34: 1206-1211.

26 Thomen RP, Walkup LL, Roach DJ, et al. Hyperpolarized ${ }^{129} \mathrm{Xe}$ for investigation of mild cystic fibrosis lung disease in pediatric patients. J Cyst Fibros 2017; 16: 275-282.

27 Walkup LL, Thomen RP, Akinyi TG, et al. Feasibility, tolerability and safety of pediatric hyperpolarized ${ }^{129} \mathrm{Xe}$ magnetic resonance imaging in healthy volunteers and children with cystic fibrosis. Pediatr Radiol 2016; 46: $1651-1662$

28 Woodhouse N, Wild JM, van Beek EJ, et al. Assessment of hyperpolarized ${ }^{3} \mathrm{He}$ lung MRI for regional evaluation of interventional therapy: a pilot study in pediatric cystic fibrosis. J Magn Reson Imaging 2009; 30: 981-988.

29 Hughes PJC, Horn FC, Collier GJ, et al. Spatial fuzzy c-means thresholding for semiautomated calculation of percentage lung ventilated volume from hyperpolarized gas and ${ }^{1} \mathrm{H}$ MRI. J Magn Reson Imaging 2018; 47: 640-646.

30 Horsley AR, Gustafsson PM, Macleod KA, et al. Lung clearance index is a sensitive, repeatable and practical measure of airways disease in adults with cystic fibrosis. Thorax 2008; 63: 135-140.

31 Smith LJ, Macleod KA, Collier GJ, et al. Supine posture changes lung volumes and increases ventilation heterogeneity in cystic fibrosis. PLoS One 2017; 12: e0188275.

32 Miller MR, Hankinson J, Brusasco V, et al. Standardisation of spirometry. Eur Respir J 2005; 26: 319-338.

33 Wanger J, Clausen JL, Coates A, et al. Standardisation of the measurement of lung volumes. Eur Respir J 2005; 26: 319-338.

34 Quanjer PH, Stanojevic S, Cole TJ, et al. Multi-ethnic reference values for spirometry for the 3-95-yr age range: the global lung function 2012 equations. Eur Respir J 2012; 40: 1324-1343.

35 Bland M. An introduction to medical statistics. In: An Introduction to Medical Statistics. Oxford, Oxford University Press, 2015; p. 124

36 Koumellis P, van Beek EJ, Woodhouse N, et al. Quantitative analysis of regional airways obstruction using dynamic hyperpolarized ${ }^{3} \mathrm{He}$ MRI-preliminary results in children with cystic fibrosis. J Magn Reson Imaging 2005; 22: $420-426$. 
Bannier E, Cieslar K, Mosbah K, et al. Hyperpolarized ${ }^{3} \mathrm{He} \mathrm{MR}$ for sensitive imaging of ventilation function and treatment efficiency in young cystic fibrosis patients with normal lung function. Radiology 2010; 255: $225-232$.

38 Altes TA, Powers PL, Knight-Scott J, et al. Hyperpolarized ${ }^{3} \mathrm{He}$ MR lung ventilation imaging in asthmatics: preliminary findings. J Magn Reson Imaging 2001; 13: 378-384.

39 de Lange EE, Altes TA, Patrie JT, et al. The variability of regional airflow obstruction within the lungs of patients with asthma: assessment with hyperpolarized helium-3 magnetic resonance imaging. J Allergy Clin Immunol 2007; 119: $1072-1078$.

40 Horsley AR, Macleod KA, Robson AG, et al. Effects of cystic fibrosis lung disease on gas mixing indices derived from alveolar slope analysis. Respir Physiol Neurobiol 2008; 162: 197-203.

41 Stewart NJ, Chan HF, Hughes PJC, et al. Comparison of ${ }^{3} \mathrm{He}$ and ${ }^{129} \mathrm{Xe}$ MRI for evaluation of lung microstructure and ventilation at 1.5T. J Magn Reson Imaging 2018; in press [https://doi.org/10.1002/jmri.25992]. 